\title{
POLYNOMIAL ROOTS FROM COMPANION MATRIX EIGENVALUES
}

\author{
ALAN EDELMAN AND H. MURAKAMI
}

\begin{abstract}
In classical linear algebra, the eigenvalues of a matrix are sometimes defined as the roots of the characteristic polynomial. An algorithm to compute the roots of a polynomial by computing the eigenvalues of the corresponding companion matrix turns the tables on the usual definition. We derive a firstorder error analysis of this algorithm that sheds light on both the underlying geometry of the problem as well as the numerical error of the algorithm. Our error analysis expands on work by Van Dooren and Dewilde in that it states that the algorithm is backward normwise stable in a sense that must be defined carefully. Regarding the stronger concept of a small componentwise backward error, our analysis predicts a small such error on a test suite of eight random polynomials suggested by Toh and Trefethen. However, we construct examples for which a small componentwise relative backward error is neither predicted nor obtained in practice. We extend our results to polynomial matrices, where the result is essentially the same, but the geometry becomes more complicated.
\end{abstract}

\section{INTRODUCTION}

Computing roots of polynomials may be posed as an eigenvalue problem by forming the companion matrix. The eigenvalues of this matrix may be found by computing the eigenvalues of this nonsymmetric matrix using standard versions of balancing (very important for accuracy!) [7] followed by the QR algorithm as may be found in LAPACK or its precursor EISPACK. This is how the MATLAB command roots performs its computation.

As Cleve Moler has pointed out in [6], this method may not be the best possible because

it uses order $n^{2}$ storage and order $n^{3}$ time. An algorithm designed specifically for polynomial roots might use order $n$ storage and $n^{2}$ time. And the roundoff errors introduced correspond to perturbations in the elements of the companion matrix, not the polynomial coefficients.

Moler continues by pointing out that

Received by the editor February 17, 1993 and, in revised form, January 10, 1994.

1991 Mathematics Subject Classification. Primary 65H05, 65F15.

Key words and phrases. Algebraic equation, QR method, eigenvalue.

The first author was supported by NSF grant DMS-9120852 and the Applied Mathematical Sciences subprogram of the Office of Energy Research, U.S. Department of Energy under Contract DE-AC03-76SF00098. 
We don't know of any cases where the computed roots are not the exact roots of a slightly perturbed polynomial, but such cases might exist.

This paper investigates whether such cases might exist. Let $\hat{r}_{i}, i=1, \ldots, n$, denote the roots of $p(x)=a_{0}+a_{1} x+\cdots+a_{n-1} x^{n-1}+x^{n}$ that are computed by this method. Further assume that the $\hat{r}_{i}$ are the exact roots of

$$
\hat{p}(x)=\hat{a}_{0}+\hat{a}_{1} x+\cdots+\hat{a}_{n-1} x^{n-1}+x^{n} .
$$

What does it mean to say that $\hat{p}$ is a slight perturbation of $p$ ? We give four answers, the best one is saved for last. In the definitions, $O(\varepsilon)$ is meant to signify a small though unspecified multiple of machine precision.

1. The "calculus" definition would require that first-order perturbations of the matrix lead to first-order perturbations of the coefficients.

2. A normwise answer that is compatible with standard eigenvalue backward error analyses is to require that

$$
\max \left|a_{i}-\hat{a}_{i}\right|=O(\varepsilon)\|C\|,
$$

where $C$ denotes the companion matrix corresponding to $p$, and $\varepsilon$ denotes the machine precision.

3. A second normwise answer that would be even better is to require that

$$
\max \left|a_{i}-\hat{a}_{i}\right|=O(\varepsilon) \| \text { balance }(C) \| .
$$

Standard algorithms for eigenvalue computations balance a matrix $C$ by finding a diagonal matrix $T$ such that $B=T^{-1} C T$ has a smaller norm than $C$.

4. The strongest requirement should be that

$$
\max \frac{\left|a_{i}-\hat{a}_{i}\right|}{\left|a_{i}\right|}=O(\varepsilon) .
$$

This is what we will mean by a small componentwise perturbation. If $a_{i}=0$, then one often wants $\hat{a}_{i}$ to be 0 too, i.e., ideally one preserves the sparsity structure of the problem.

It was already shown by Van Dooren and Dewilde [11, p. 576] by a block Gaussian elimination argument that the calculus definition holds. Their argument is valid in the more complicated case of polynomial matrices. It immediately follows that good normwise answers are available, though it is not clear what exactly are the constants involved. We found that integrating work by Arnold [1] with Newton-like identities allows for an illuminating geometrical derivation of a backward error bound that is precise to first order. Our work improves on [11] in that we derive the exact first-order perturbation expression which we test against numerical experiments. Numerical experiments by Toh and Trefethen [9] compare this algorithm with the Jenkins-Traub or the Madsen-Reid algorithm. These experiments indicate that all three algorithms have roughly similar stability properties and further that there appears to be a close link between the pseudospectra of the balanced companion matrix and the pseudozero sets of the corresponding polynomial. 


\section{ERROR ANALYSIS}

2.1. Problem statement and solution. Let $p(z)=a_{0}+a_{1} z+\cdots+a_{n-1} z^{n-1}+z^{n}$ be any monic polynomial. The companion matrix of this polynomial,

$$
C=\left(\begin{array}{ccccc}
0 & & & & -a_{0} \\
1 & 0 & & & -a_{1} \\
& 1 & 0 & & -a_{2} \\
& & \ddots & & \vdots \\
& & & 1 & -a_{n-1}
\end{array}\right),
$$

has characteristic polynomial $P_{C}(z) \equiv \operatorname{det}(z I-C)=p(z)$.

If $E$ is a dense perturbation matrix with "small" entries, the natural error analysis question is the computation of $P_{C+E}(z)-P_{C}(z) \equiv \delta a_{0}+\delta a_{1} z+\cdots+$ $\delta a_{n-1} z^{n-1}$. In MATLAB style notation, we are studying poly $(C+E)-\operatorname{poly}(C)$ to first order.

Our result is

Theorem 2.1. To first order, the coefficient of $z^{k-1}$ in $P_{C+E}(z)-P_{C}(z)$ is

$$
\sum_{m=0}^{k-1} a_{m} \sum_{i=k+1}^{n} E_{i, i+m-k}-\sum_{m=k}^{n} a_{m} \sum_{i=1}^{k} E_{i, i+m-k},
$$

where $a_{n}$ is defined to be 1 .

In particular, we see that a small perturbation $E$ introduces errors in the coefficients that are linear in the $E_{i j}$. Since it is well known that standard eigenvalue procedures compute eigenvalues of matrices with a "small" backward error, we have a precise sense in which we claim that there is a polynomial near $P_{C}(z)$ whose exact roots are computed in this manner.

For convenience, we state this result in a matrix times vector format. Let

$$
f_{k, d} \equiv \sum_{i=1}^{k} E_{i, i+d} \quad \text { and } \quad b_{k, d} \equiv \sum_{i=k}^{n} E_{i, i+d} .
$$

These are the forward and backward "plus-scans" or "prefixes" of the $d$ th diagonal of $E$. Our result is that the matrix-vector product (3)

$$
\begin{aligned}
\left(\begin{array}{c}
\delta a_{0} \\
\delta a_{1} \\
\delta a_{2} \\
\vdots \\
\delta a_{n-1}
\end{array}\right)= & \left(\begin{array}{ccccccc}
b_{2,-1} & -f_{1,0} & -f_{1,1} & \cdots & -f_{1, n-3} & -f_{1, n-2} & -f_{1, n-1} \\
b_{3,-2} & b_{3,-1} & -f_{2,0} & \cdots & -f_{2, n-4} & -f_{2 n-3} & -f_{2, n-2} \\
\vdots & \vdots & \vdots & \ddots & \vdots & \vdots & \vdots \\
b_{n,-(n-1)} & b_{n,-(n-2)} & b_{n,-(n-3)} & \cdots & b_{n,-1} & -f_{n-1,0} & -f_{n-1,1} \\
0 & 0 & 0 & \cdots & 0 & 0 & -f_{n, 0}
\end{array}\right) \\
& \cdot\left(\begin{array}{c}
a_{0} \\
a_{1} \\
a_{2} \\
\vdots \\
a_{n-1} \\
a_{n=1}=
\end{array}\right)
\end{aligned}
$$

is correct to first order. The $n \times(n+1)$ matrix above contains backward prefixes in the lower triangular part and forward prefixes in the upper triangular part. 
The last row of the matrix equation states that perturbing the trace of a matrix perturbs the (negative of the) coefficient of $z^{n-1}$ by the same amount.

If we further assume that the $E$ is the backward error matrix computed by a standard eigenvalue routine, then we might as well assume that $E$ is nearly upper triangular. There will also be elements on the subdiagonal, and possibly on the next lower diagonal, depending on exactly which eigenvalue method is used.

A result analogous to Theorem 2.1 for matrix polynomials may be found in $\S 4$.

2.2. Geometry of tangent spaces and transversality. Figure 1 illustrates matrix space $\left(R^{n \times n}\right)$ with the usual Frobenius inner product

$$
(A, B) \equiv \operatorname{tr}\left(A B^{T}\right)
$$

The basic players are

$$
\begin{array}{ll}
p(z) & \text { a polynomial (not shown) } \\
C & \text { corresponding companion matrix } \\
\text { Orb } & \text { manifold of matrices similar to } C \\
\text { Tan } & \text { tangent space to this manifold }=\left\{C X-X C: X \in R^{n \times n}\right\} \\
\text { Normal } & \text { normal space to this manifold }=\left\{q\left(C^{T}\right): q \text { is a polynomial }\right\} \\
\text { Syl } & \text { "Sylvester" family of companion matrices }
\end{array}
$$

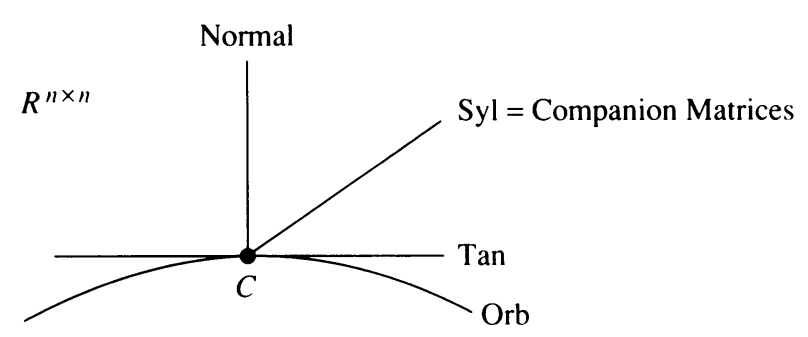

FIGURE 1. Matrix space near a companion matrix

- The curved surface represents the orbit of matrices similar to $C$. These are all the nonderogatory matrices that have eigenvalues equal to the roots of $p$ with the same multiplicities. (The dimension of this space turns out to be $n^{2}-n$. An informal argument is that only $n$ parameters are specified, i.e., the eigenvalues.)

- The tangent space to this manifold, familiar to anyone who has studied elementary Lie algebra (or performed a first-order calculation), is the set of commutators $\left\{C X-X C: X \in R^{n \times n}\right\}$. (Dimension $=n^{2}-n$.)

- It is easy to check that if $q$ is a polynomial, then any matrix of the form $q\left(C^{T}\right)$ is perpendicular to every matrix of the form $C X-X C$. Only slightly 
more difficult [1] is to verify that all matrices in the normal space are of the form $q\left(C^{T}\right)$. (Dimension $=n$.)

- The set of companion matrices (also known as the "Sylvester" family) is obviously an affine space through $C .($ Dimension $=n$. $)$

Proposition 2.1. The Sylvester space of companion matrices is transversal to the tangent space; i.e., every matrix may be expressed as a linear combination of a companion matrix and a matrix in the tangent space.

This fact may be found in [1]. When we resolve $E$ into components in these two directions, the former displays the change in the coefficients (to first order) and the latter does not affect the coefficients (to first order). Actually, a stronger statement holds: the resolution is unique. In the language of singularity theory, not only do we have transversality, but also universality: unique + transversal . We explicitly perform the resolution, and thereby prove the proposition, in the next subsection.

In numerical analysis, there are many examples where perburbations in "nonphysical" directions cause numerical instability. In the companion matrix problem, only perturbations to the polynomial coefficients are relevant to the problem. Other perturbations are by-products of our numerical method. It is fortunate in this case that the error produced by an entire $\left(n^{2}-n\right)$-dimensional space of extraneous directions is absorbed by the tangent space.

2.3. Algebra-the centralizer. Let us take a close look at the centralizer of $C$. This is the $n$-dimensional linear space of polynomials in $C$, because $C$ is nonderogatory. Taking the $a_{k}$ as in (1), for $k=0, \ldots, n-1$, and letting $a_{n}=1, a_{j}=0$ for $j \notin[0, n]$, we define matrices

$$
M_{k}=\sum_{j=k}^{n} a_{j} C^{j-k} .
$$

Clearly, $M_{0}=0, M_{n}=I$, and the $M_{k}, k=1, \ldots, n$, span the centralizer of C.

An interesting relationship (which is closely related to synthetic division) is that

$$
p(t)(t-C I)^{-1}=\sum_{k=1}^{n} M_{k} t^{k-1}
$$

[2, p. 85 , equation (32)]. The trace of the above equation is the Newton identities in generating function form; a variation on the above equation gives the exact inverse of a Vandermonde matrix [10].

A more important relationship for our purposes is that

$$
M_{k}=C M_{k+1}+a_{k} I, \quad k=0,1,2, \ldots,
$$

from which we can inductively prove (the easiest way is backwards from $k=n$ ) that 


$$
M_{k}=\left(\begin{array}{cccc|ccc}
\multicolumn{2}{c}{\underline{k} a_{k}} & & & & & \\
a_{k+1} & a_{k} & & & & & \\
-a_{1} & \ddots & \\
\vdots & a_{k+1} & \ddots & & \vdots & \ddots & -a_{0} \\
a_{n}=1 & \vdots & \ddots & a_{k} & -a_{k-1} & \ddots & \vdots \\
& a_{n}=1 & \ddots & a_{k+1} & & \ddots & \vdots \\
& & \ddots & \vdots & & & -a_{k-1}
\end{array}\right) .
$$

This is almost the Toeplitz matrix with $(i, j)$ entry equal to $\pm a_{k+i-j}$ except that the left side of the matrix is lower triangular and the right side is strictly upper triangular.

We are now ready to resolve any $E$ into

$$
E=E^{\text {tan }}+E^{\text {syl }} .
$$

All we need is the relationship that expresses how $E^{\text {tan }}$ is perpendicular to the normal space:

$$
\operatorname{tr}\left(M_{k} E^{\tan }\right)=0 \text { for } k=1, \ldots, n .
$$

We therefore conclude from (4) that

$$
\operatorname{tr}\left(M_{k} E\right)=\operatorname{tr}\left(M_{k} E^{\text {syl }}\right)=E_{k, n}^{\text {syl }} .
$$

Because of the almost Toeplitz nature of the $M_{k}$, the trace of $M_{k} E$ involves partial sums of elements of $E$ along certain diagonals. Writing out this trace, we have that

$$
-E_{k, n}^{\mathrm{syl}}=\sum_{m=0}^{k-1} a_{m} \sum_{i=k+1}^{n} E_{i, i+m-k}-\sum_{m=k}^{n} a_{m} \sum_{i=1}^{k} E_{i, i+m-k} .
$$

The above expression gives the coefficient of the perturbed characteristic polynomial correct to first order. (Since the coefficients are negated in (1), we are interested in $-E_{k, n}^{\text {syl }}$.)

Since $E^{\text {syl }}$ is zero in every column other than the last, we may also use (4) to calculate $E^{\text {tan }}$, should we choose.

\section{NUMERICAL EXPERIMENTS}

3.1. A pair of $2 \times 2$ examples. The companion matrices

$$
A=\left(\begin{array}{cc}
0 & -1 \\
1 & 2^{k}
\end{array}\right) \quad \text { and } B=\left(\begin{array}{cc}
0 & 1 \\
1 & 2^{-3 k}
\end{array}\right)
$$

for $k$ not too small illustrate many subtleties that occur in floating-point arithmetic. For convenience, we assume our arithmetic follows the IEEE doubleprecision standard, for which $k=27$ is large enough to illustrate our point. 
To machine accuracy, the eigenvalues of $A$ are $2^{k}$ and $2^{-k}$. The eigenvalues of $B$ are 1 and -1 . LAPACK and MATLAB compute $2^{k}$ and 0 for the eigenvalues of $A$, while the eigenvalues of $B$ are computed to be $1-2^{-52}$ and -1 . Neither of these matrices is affected by balancing. Both of these answers are consistent with the error estimate in (2). Neither of these matrices gives answers with a small componentwise backward error. In the first case, the given product of the roots is 1 while the exact product of the computed roots is 0 . In the second case, the given sum of the roots is $2^{-81}$, while the exact sum of the computed roots is $-2^{-52}$.

However, MATLAB and LAPACK could be more accurate! Both packages compute the Schur form of a $2 \times 2$ matrix using an algorithm that is more unstable for the smaller eigenvalue than is necessary. We propose that such high-quality packages should compute the eigenvalues of a general $2 \times 2$ matrix by solving the quadratic equation as accurately as possible, given the rounded values of the trace and the determinant. If we have a $2 \times 2$ companion matrix, then there will be no roundoff error in the trace and the determinant.

The lesson of these examples is that the roots could be computed far more accurately than would be predicted by our error bound (2), but currently LAPACK and MATLAB return eigenvalues that are consistent with our bound. The other lesson is that without further assumptions it is impossible to require a small componentwise backward error. Fortunately, these examples are rather pathological. As we will see in the next subsection, in practice we do expect to compute roots with a small componentwise backward error.

3.2. A more "realistic" set of tests. In this subsection we use Theorem 2.1 to predict the componentwise backward error. We also perform numerical experiments to measure the componentwise backward error. Our results show that Theorem 2.1 always predicts a small backward error and is at most only pessimistic by one, two, or maybe three digits.

To predict the error, we must model the backward error introduced by the $\mathrm{QR}$ algorithm. We decided to choose a model that is designed to compensate for pessimistic factors often found in rounding error analyses. Therefore, the model does not provide a rigorous bound, but at least in our test cases it seems to work well in practice.

What we do is consider an error matrix $E$ with entries $\varepsilon=2^{-52}$ in all entries $(i, j)$ with $j-i \geq-2$. For example, when $n=6$,

$$
E=\left(\begin{array}{llllll}
\varepsilon & \varepsilon & \varepsilon & \varepsilon & \varepsilon & \varepsilon \\
\varepsilon & \varepsilon & \varepsilon & \varepsilon & \varepsilon & \varepsilon \\
\varepsilon & \varepsilon & \varepsilon & \varepsilon & \varepsilon & \varepsilon \\
0 & \varepsilon & \varepsilon & \varepsilon & \varepsilon & \varepsilon \\
0 & 0 & \varepsilon & \varepsilon & \varepsilon & \varepsilon \\
0 & 0 & 0 & \varepsilon & \varepsilon & \varepsilon
\end{array}\right)
$$

This structure allows (with some overkill) for the possibility of double shifting in the eigenvalue algorithm. A dense perturbation matrix did not make a substantial difference in our test cases.

The standard algorithms balance the matrix by finding a diagonal matrix $T$ such that $B=T^{-1} A T$ has a smaller norm than $A$. Our model will be that 
the eigenvalue algorithm computes the exact eigenvalues of a matrix $B+E^{\prime}$, where $\left|E^{\prime}\right| \leq E$; i.e., each element of $E^{\prime}$ has absolute value at most $\varepsilon$ above the second lower diagonal and is 0 otherwise. Therefore, we are computing the exact eigenvalues of $A+T E^{\prime} T^{-1}$. To first order, then, the error in the coefficients is bounded by the absolute value of the matrix times the absolute value of the vector in the product (3), where the scans are computing using $T E T^{-1}$. This is how we predict the $\delta_{i}$. Further details appear in our MATLAB code in the Appendix.

Following [9] exactly, we explore the following degree-20 monic polynomials:

(1) “Wilkinson polynomial": zeros $1,2,3, \ldots, 20$,

(2) the monic polynomial with zeros equally spaced in the interval $[-2.1$, 1.9],

(3) $p(z)=(20 !) \sum_{k=0}^{20} z^{k} / k !$,

(4) the Bernoulli polynomial of degree 20 ,

(5) the polynomial $z^{20}+z^{19}+z^{18}+\cdots+1$,

(6) the monic polynomial with zeros $2^{-10}, 2^{-9}, 2^{-8}, \ldots, 2^{9}$,

(7) the Chebyshev polynomial of degree 20 ,

(8) the monic polynomial with zeros equally spaced on a sine curve, viz., $(2 \pi / 19(k+0.5))+i \sin (2 \pi / 19(k+0.5)), k=-10,-9,-8, \ldots, 9$.

Our experimental results consist of three columns for each polynomial. To be precise, we first computed the coefficients either exactly or with 30-decimal precision using Mathematica. We then read these numbers into MATLAB which may not have rounded the last bit or two correctly. ${ }^{1}$ Though we could have rounded the result correctly in Mathematica, we chose not to do so. Rather, we took the rounded polynomials stored in MATLAB to be our "official" test cases.

Column 1: Log Predicted Error: In the first column we model the predicted error from (2) in the manner we described above. First we compute the modeled $\delta a_{i}$, and then display the rounded value of $\log _{10}\left|\delta a_{i} / a_{i}\right|$.

Column 2: Log Computed Error: We computed the eigenvalues using MATLAB, and then translated the IEEE double-precision numbers to Mathematica without any error. We then computed the exact polynomial with these computed roots and compared the relative backward error in the coefficients.

Column 3: Pessimism Index: By taking the ratio of the computed error in column 2 to the predicted error described in column 1 and then taking the logarithm and rounding, we obtain a pessimism index. Indices such as $0,-1$, and -2 indicate that we are pessimistic by at most two orders of magnitude; and index of -19 indicates a pessimism of 19 orders of magnitude. (Since we are using negative numbers, perhaps we should more properly call this an optimism index.)

There are no entries where the polynomial coefficient is zero. (The Bernoulli polynomial is a little funny in that it has a $z^{19}$ term, but no other odd-degree

\footnotetext{
${ }^{1}$ Try entering
}

$1.00000000000000018,1.00000000000000019$, and 1.0000000000000001899999999

into MATLAB (fifteen zeros in each number). The results that we obtained on a SUN Sparc Station 10 were $1,1+2^{-52}$, and $1-2^{-52}$, respectively, though the correctly rounded result should be $1+2^{-52}$ in all instances. Cleve Moler has responded that a better string parser is needed. 


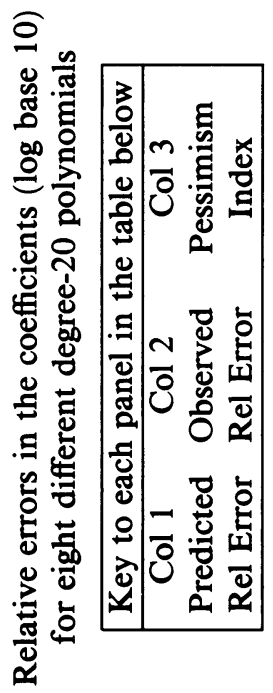

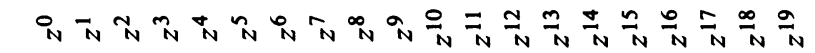

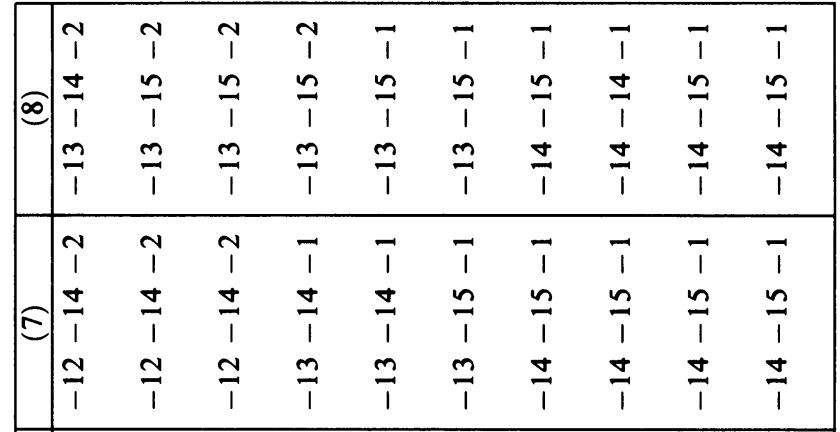

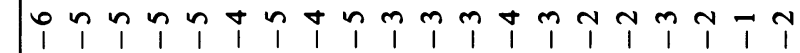

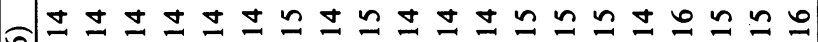
6)

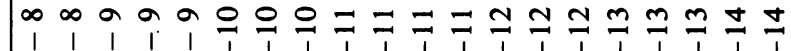

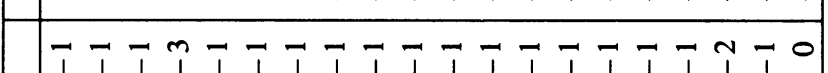

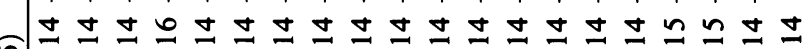

( $\begin{array}{llllllllllllllllllll}1 & 1 & 1 & 1 & 1 & 1 & 1 & 1 & 1 & 1 & 1 & 1 & 1 & 1 & 1 & 1 & 1 & 1 & 1 & 1\end{array}$

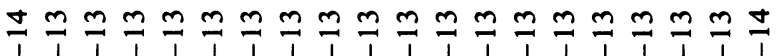

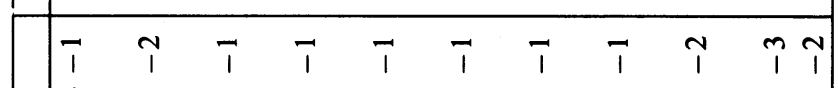

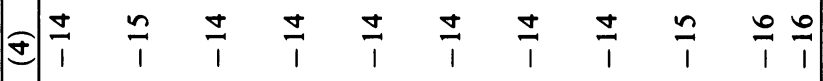

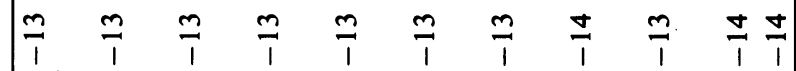

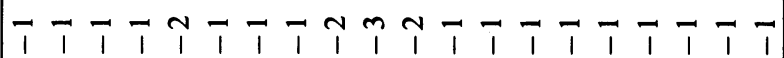

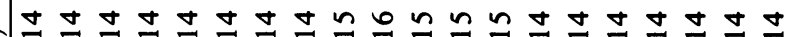
(ิ)

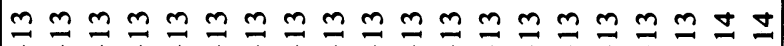

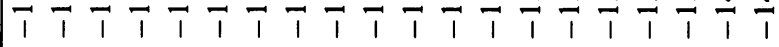

-

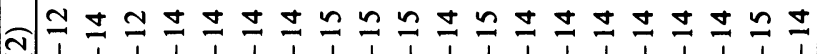

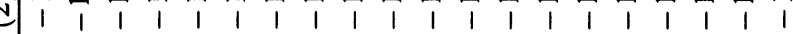

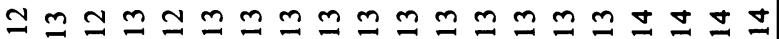

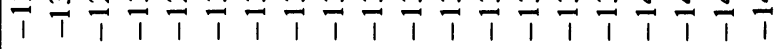

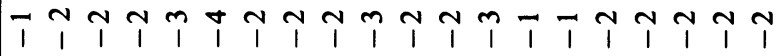

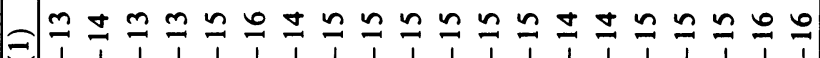

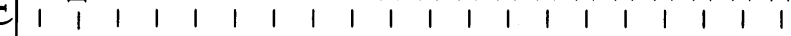

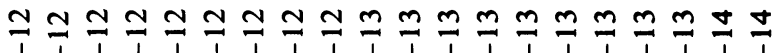
$\frac{\uparrow}{d}$ 
term.) The computed relative error would be infinite in many of these cases. The top coefficient is the log relative error in the determinant, i.e., the coefficient of the constant term; the bottom coefficient refers to the trace, i.e., the coefficient of $t^{19}$.

In all cases, we see that the computed backward relative error was excellent. With the exception of column (6), this is fairly well predicted, usually with a pessimism index of one to three orders of magnitude. Column (6) is an exception, where the backward error is far more favorable than we predict.

Why this might be possible was explained in the previous subsection. We know the determinant to full precision (very rare when performing matrix computations!). So long as our QR shifts and deflation criteria manage not to destroy the determinant, it will remain intact. In column (6) we see a case where this occurred. By contrast, in the previous subsection we illustrated a case where this did not occur.

We suspect that for any companion matrix, it is often-if not alwayspossible to choose shifts and deflation criteria to guarantee high (backward relative) accuracy even with the smallest of determinants, but we have not proven this.

\section{Generalization to MATRiX POlynomials}

A monic matrix polynomial has the form

$$
P(x)=A_{0}+A_{1} x+\cdots+A_{n-1} x^{n-1}+I_{p} x^{n},
$$

where we assume that the coefficients $A_{i}$ (and $A_{n} \equiv I_{p}$ ) are $p \times p$ matrices, and $x$ is a scalar. It is of interest $[3,4,5]$ to find the $x$ for which $\operatorname{det}(P(x))=$ 0 and the corresponding eigenvectors $v(x)$ such that $P(x) v(x)=0$. Such information may be obtained from the $p n \times p n$ block companion matrix

$$
C=\left(\begin{array}{ccccc}
0 & & & & -A_{0} \\
I_{p} & 0 & & & -A_{1} \\
& I_{p} & 0 & & -A_{2} \\
& & \ddots & & \vdots \\
& & & I_{p} & -A_{n-1}
\end{array}\right) .
$$

The Sylvester space now has dimension $n p^{2}$, while the tangent space to the orbit of $C$ generically has dimension $n^{2} p^{2}-n p$, though it can be smaller. It seems that if $p>1$, we have too many dimensions! We will now show that we may proceed in a manner that is analogous to that of $\S 2.3$ to obtain what is roughly the same answer, but to do so we must carefully pick a natural subspace of the tangent space that will give a universal decomposition. This is not necessary when $p=1$. The natural subspace of the tangent space consists of all matrices of the form $C X-X C$ where the last $p$ rows of $X$ are 0 .

Lemma 4.1. Define

$$
M_{k} \equiv \sum_{j=k}^{n} C^{j-k}\left(I_{n} \otimes A_{j}\right)
$$


where $\otimes$ denotes the Kronecker product and $I_{n}$ is the identity matrix of order $n$. Then

$$
M_{k}=C M_{k+1}+I_{n} \otimes A_{k}
$$

and

$$
M_{k}=\left(\begin{array}{cccc|ccc}
\multicolumn{2}{c}{\underline{k}} & & & & \multicolumn{3}{c}{\frac{n-k}{2}} & \\
A_{k} & & & & -A_{0} & & \\
A_{k+1} & A_{k} & & & -A_{1} & \ddots & \\
\vdots & A_{k+1} & \ddots & & \vdots & \ddots & -A_{0} \\
A_{n}=I_{p} & \vdots & \ddots & A_{k} & -A_{k-1} & \ddots & \vdots \\
& A_{n}=I_{p} & \ddots & A_{k+1} & & \ddots & \vdots \\
& & \ddots & \vdots & & & -A_{k-1}
\end{array}\right) .
$$

Proof. These statements are readily verified by induction.

We now introduce the $p \times p$ block trace of a matrix:

Definition 4.1. If $Z$ is a $p n \times p n$ matrix whose $p \times p$ blocks are denoted $Z_{i j}$, then we define

$$
\operatorname{tr}_{p}(Z) \equiv \sum_{i=1}^{n} Z_{i i}
$$

Notice that $\operatorname{tr}_{p}(Z)$ is a $p \times p$ matrix, not a scalar.

Theorem 4.1. Given the first $n-1$ block columns of a pn $\times p n$ matrix $Z$, the condition that

$$
0=\operatorname{tr}_{p}\left(Z M_{k}\right), \quad k=0, \ldots, n,
$$

is equivalent to the condition that

$$
Z=C X-X C \text { for some } X \text { with } 0 \text { bottom block row. }
$$

Moreover, either condition determines the final block column of $Z$ uniquely, given the remaining columns.

Proof. The $(n, k)$ block entry of $M_{k}$ is $I_{p}$, and this determines $Z_{k n}$ uniquely from (7). If $X$ has 0 as its bottom block row, it is easy to verify that the map from $X$ to the first $n-1$ block columns of $C X-X C$ has a trivial nullspace. Thus, $Z$ is uniquely determined by $(8)$.

What remains is to show (8) implies (7). Suppose that $Y$ has a zero bottom block row. Then $\operatorname{tr}_{p}(C Y)=\operatorname{tr}_{p}(Y C)=\sum_{i=1}^{n-1} Y_{i, i+1}$. Therefore, if $X$ has a zero bottom block row, then $\operatorname{tr}_{p}\left(C X M_{k}\right)=\operatorname{tr}_{p}\left(X M_{k} C\right)$ by choosing $Y=X M_{k}$. Finally $\operatorname{tr}_{p}\left(X C^{j}\left(I_{n} \otimes A\right) C-X C^{j} C\left(I_{n} \otimes A\right)\right)=0$ for any $p \times p$ matrix $A$, because $\left(I_{n} \otimes A\right) C-C\left(I_{n} \otimes A\right)$ is 0 except for ihe last block column. Therefore, $X M_{k} C=X C M_{k}$, from which we conclude that $\operatorname{tr}_{p}\left(C X M_{k}\right)=\operatorname{tr}_{p}\left(X M_{k} C\right)=$ $\operatorname{tr}_{p}\left(X C M_{k}\right)$. 
We now summarize the geometry.

Corollary 4.1. In the $\left(n^{2} p^{2}\right)$-dimensional space of $n p \times n p$ matrices, the $n^{2} p^{2}-$ $n p^{2}$ subspace of the tangent space of the orbit of $C$ defined either by (7) or (8) is transversal at $C$ to the $\left(n p^{2}\right)$-dimensional Sylvester space consisting of block companion matrices.

We may now resolve any perturbation matrix $E$ into

$$
E=E^{\text {tan }}+E^{\text {syl }}
$$

as in (4), except now $E^{\text {tan }}$ must be in this $\left(n^{2} p^{2}-n p^{2}\right)$-dimensional subspace, and $E^{\text {syl }}$ is 0 except in the final block column. Because $\operatorname{tr}_{p}\left(Z M_{k}\right) \neq \operatorname{tr}_{p}\left(M_{k} Z\right)$, the correct result is that

$$
-E_{k, n}^{\mathrm{syl}}=\sum_{m=0}^{k-1}\left(\sum_{i=k+1}^{n} E_{i, i+m-k}\right) A_{m}-\sum_{m=k}^{n}\left(\sum_{i=1}^{k} E_{i, i+m-k}\right) A_{m} .
$$

The above expression gives the block coefficient of a perturbed matrix polynomial correct to first order.

\section{ACKNOWLEDGMENTS}

We would like to thank Jim Demmel for many interesting discussions and experiments on transversality and polynomial rootfinding. 


\section{APPENDIX}

MATLAB program used in experiments. The numerical part of our experiments was performed in MATLAB, while the exact component of the experiments was performed with Mathematica. We reproduce only the main MATLAB code below for the purpose of specifying precisely the crucial components of our experiments. The subroutines scan and scanr compute the plus-scan and the reversed plus-scan of a vector, respectively.

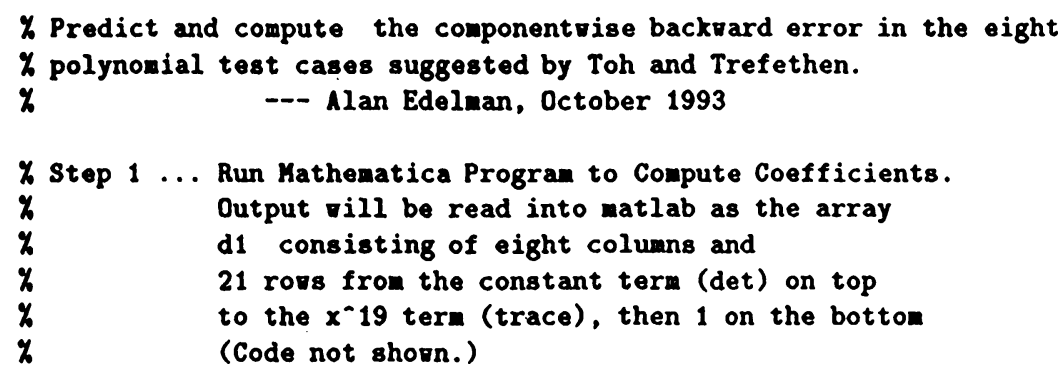




\section{BIBLIOGRAPHY}

1. V. I. Arnold, On matrices depending on parameters, Russian Math. Surveys 26 (1971), 29-43.

2. F. R. Gantmacher, The theory of matrices, Vols. 1, 2, Chelsea, New York, 1959.

3. I. Gohberg, P. Lancaster, and L. Rodman, Matrix polynomials, Academic Press, New York, 1982.

4. D. Manocha and J. Demmel, Algorithms for intersecting parametric and algebraic curves I: simple intersections, ACM Trans. Graphics 13 (1994), 73-100.

5. __ Algorithms for intersecting parametric and algebraic curves II: multiple intersections, Computer graphics, vision and image processing: Graphical models and image processing (to appear).

6. C. Moler, Cleve's corner: ROOTS-Of polynomials, that is, The MathWorks Newsletter, vol. 5, no. 1 (Spring 1991), 8-9.

7. B. Parlett and C. Reinsch, Balancing a matrix for calculation of eigenvalues and eigenvectors, Numer. Math. 13 (1969), 293-304.

8. W. H. Press, B. P. Flannery, S. A. Teukolsky, and W. T. Vetterling, Numerical recipes-The art of scientific computing, Cambridge Univ. Press, Cambridge, 1986.

9. K.-C. Toh and L. N. Trefethen, Pseudozeros of polynomials and pseudospectra of companion matrices, Numer. Math. 68 (1994), 403-425.

10. J. F. Traub, Associated polynomials and uniform methods for the solution of linear problems, SIAM Rev. 8 (1966), 277-301.

11. P. Van Dooren and P. Dewilde, The eigenstructure of an arbitrary polynomial matrix: computational aspects, Linear Algebra Appl. 50 (1983), 545-579.

Department of Mathematics, Massachusetts Institute of Technology, Cambridge, MASSACHUSETTS 02139

E-mail address: edelman@math.mit.edu

Quantum Chemistry laboratory, Department of Chemistry, Hokkaido University, SAPPORO 060, JAPAN

E-mail address: hiroshi@chem2.hokudai.ac.jp 